\title{
Research Paper \\ Lifestyle of Elderly People With Osteoporosis and Its Related Factors
}

\author{
*Esmaeil Fallah Mehrabadi ${ }^{1}$, Minoo Pakgohar ${ }^{1}$, Sedigheh Asadi², Hamid Haghani ${ }^{3}$
}

1. Department of Geriatric Nursing, School of Nursing and Midwifery, Tehran University of Medical Sciences, Tehran, Iran.

2. Department of Health Management and Economics, School of Public Health, Tehran University of Medical Sciences, Tehran, Iran

3. Department of Biostatistics, School of Public Health, Iran University of Medical Sciences, Tehran, Iran.

Received: 30 Jan. 2017 Accepted: 21 May 2017

Keywords:

Lifestyle, Osteopo-

rosis, Elderly people
Chtation: Fallah Mehrabadi E, Pakgohar M, Asadi S, Haghani H. [Lifestyle of Elderly People With Osteoporosis and Its Related Factors (Persian)]. Iranian Journal of Ageing. 2017; 12(2):132-145. http://dx.doi.org/10.21859/sija-1202132

http://dx.doi.org/10.21859/sija-1202132

\begin{abstract}
A B S T R A C T
Objectives Given the aging process of the population and the important role of lifestyle in common and chronic diseases, especially osteoporosis in the elderly, this study was conducted to evaluate the lifestyle of elderly people with osteoporosis.

Methods \& Materials This cross sectional (descriptive-analytic) study was carried out in 2013 on 300 osteoporotic elderly people who were referred to the bone densitometry centers of Tehran University of Medical Sciences, Tehran, Iran. Convenience sampling method was used, and the data were collected using a questionnaire for measuring healthy lifestyle in Iranian elderly. The collected data were analyzed by descriptive statistics including ANOVA and t-test by using SPSS-PC (V. 21) and P $\leq 0.05$.

Results Results showed that the lifestyle of most osteoporotic elderly people (71.3\%) was moderate, $10.7 \%$ of them had a desirable lifestyle, and $18 \%$ had an undesirable lifestyle. There was no statistically significant difference between the overall average score of lifestyle with age $(P=0.499)$ and sex $(P=0.176)$ in older adults with osteoporosis, but significant difference was statistically observed between the overall average score of lifestyle and marital status $(P=0.001)$, educational level $(P=0.027)$, and chronic disease $(\mathrm{P}=0.009)$.

Conclusion Due to the increasing elderly population and the prevalence of osteoporosis in them, it is recommended that health officials and policy makers in the country should pay more attention in this area to prevent the occurrence of the disease as well as improve the lifestyle of elderly people with osteoporosis.
\end{abstract}

\section{Extended Abstract}

\section{Objectives}

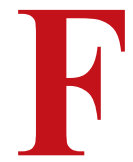

ailure to adhere to a healthy lifestyle leads to increased mortality [1]. In addition, a healthy lifestyle can help prevent chronic and common diseases of aging such as osteoporosis. Therefore, evaluating the lifestyle of the elderly with osteoporosis is of great importance in order to prevent diseases and improve their quality of life and health status [2].

The aging population of Iran and, most importantly, the role of lifestyle in chronic diseases of the elderly, especially those with osteoporosis, have been considered while designing the study. As lifestyle is dependent on the culture of the people and the lifestyle of the Iranian commu-

* Corresponding Author:

Esmaeil Fallah Mehrabadi, MSc.

Address: Department of Geriatric Nursing, School of Nursing and Midwifery, Tehran University of Medical Sciences, Tehran, Iran.

Tel: +98 (939) 3931993

E-mail: fallahmehrabadi.e@gmail.com 
nity is different from that of the other countries (3.4), the current study aimed to assess the lifestyle of the elderly with osteoporosis in Iran.

\section{Methods \& Materials}

This study is a cross-sectional descriptive-analytic study that has been conducted on 300 elderly people with osteoporosis, using available sampling method. Data was collected using a healthy lifestyle measurement questionnaire among Iranian elderly people. This questionnaire was prepared by Ishaghi et al. [5] in Isfahan University of Medical Sciences and has 46 questions in the area of prevention (15 items); physical activity, exercise, and recreation (5 items); healthy nutrition (14 questions); stress management (5 questions); and social and interpersonal relationships (7 questions). Each question is rated on a scale of 5. Score 1 represents the most unfavorable lifestyle, and the increasing scores of 2, 3, 4 and 5 mean increasing utility level of lifestyle. Thus, the lowest achievable score through the questionnaire is 42 , and the highest score is 211 . Eventually, based on acquired scores, lifestyle is divided into three levels: undesirable lifestyle (score 42-98), medium lifestyle (99-155), and the ideal lifestyle (156-211) [19]. Validity and reliability of the questionnaire has been measured previously by Ishaghi et al. To determine the validity of the questionnaire, comments and judgment from experts and also the target group, which included elderly people, were used, and for the reliability of the questionnaire, the Cronbach's Alpha Method was determined to be 0.76 [19]. Data analysis is performed using SPSS software (V. 21) and statistical t-test. Analysis of variance and descriptive statistics were performed $(\mathrm{P} \leq 0.05)$. This study has been confirmed by the Ethics Committee of Tehran University of Medical Sciences with letter No. 903/130/D/92, and all of the 22 codes of considerations and ethics are observed in this research.

\section{Results}

The majority of participants in this study (68.4\%) were in the age range of 60 to 70 years, and the mean age of the subjects studied is 67 years with a standard deviation of 5.63 . In the study, $62.1 \%$ of the elderly were female, $73.3 \%$ were married, $54 \%$ had elementary education, and $94 \%$ of the elderly were literate. In terms of body mass index , the majority ( $43 \%$ ) of research subjects were overweight with a mean of $26.26 \pm 3.78$. In total, $67.3 \%$ of elderly people present in the study suffered from a chronic illness other than osteoporosis.

Results in the prevention area, 52\% of participants with a mean and standard deviation of $77.51 \pm 7.07$, in the healthy diet $85 \%$ with an average and standard deviation of $32.86 \pm 5.86$, in the area of stress management $82.3 \%$ with an average and standard deviation of $14.18 \pm 2.66$ and in terms of interpersonal and social relations $72.6 \%$ with a mean and standard deviation of $21.73 \pm 4.15$ have a moderate lifestyle. Also in the field of sports, recreation and entertainment, $52.6 \%$ of participants with the mean and standard deviation of $10.89 \pm 3.16$ have an unfavorable lifestyle. The majority (71.3\%) of elderly people with osteoporosis in the present study has an average lifestyle, $10.7 \%$ have a favorable lifestyle, and 18\% have an unfavorable lifestyle.

Research results show that majority of participants in the research in all three age groups of 60-70, 71-80, and over 80 years had a medium lifestyle, and there is no significant difference between the overall average score of lifestyle and age in the elderly with osteoporosis $(\mathrm{P}=0.499)$.

T-test results (Table 1) showed that there is no significant difference between the two groups in terms of mean

Table 1. Average lifestyle score and its dimensions in research subjects based on gender

\begin{tabular}{|c|c|c|c|c|c|c|}
\hline \multicolumn{7}{|c|}{ Lifestyle Score } \\
\hline Gender & Total & Prevention & $\begin{array}{l}\text { Physical Activity of } \\
\text { Sports, Recreation and } \\
\text { Entertainment }\end{array}$ & $\begin{array}{l}\text { Healthy } \\
\text { Nutrition }\end{array}$ & Stress Management & $\begin{array}{l}\text { Social and Interper- } \\
\text { sonal Relationships }\end{array}$ \\
\hline Male & $132.46 \pm 16.27$ & $49.09 \pm 8.05$ & $11.31 \pm 3.07$ & $32.01 \pm 4.59$ & $14.76 \pm 2.35$ & $22.56 \pm 3.77$ \\
\hline Female & $129.75 \pm 17.56$ & $53.41 \pm 5.84$ & $10.63 \pm 3.19$ & $33.36 \pm 6.48$ & $13.82 \pm 2.79$ & $4.29 \pm 21.22$ \\
\hline Total & $131.43 \pm 16.80$ & $51.77 \pm 7.07$ & $10.89 \pm 3.16$ & $32.85 \pm 5.86$ & $14.18 \pm 2.66$ & $21.73 \pm 4.15$ \\
\hline \multirow{3}{*}{ T-test } & $P<0.176$ & $P<0.001$ & $P<0.072$ & $P<0.054$ & $P<0.003$ & $P<0.006$ \\
\hline & $t=-1.357$ & $t=-5.361$ & $t=-1.805$ & $t=-1.933$ & $t=2.948$ & $t=2.745$ \\
\hline & $d f=298$ & $\mathrm{df}=298$ & $d f=298$ & $d f=298$ & $d f=298$ & $d f=298$ \\
\hline
\end{tabular}


score of overall lifestyle in the elderly taking into account the gender $(\mathrm{P}=0.176)$.

In the present study, marital status has been identified as a factor associated with lifestyle in elderly people with osteoporosis. ANOVA test results showed that there is a significant difference between the two groups in terms of mean score of lifestyle in the elderly taking into account their marital status $(\mathrm{P}=0.001)$. Group of widows and singles had a lower mean score of lifestyle as compared to the married ones.

Results showed that with increasing levels of education, the lifestyle mean score of participants increased; based on the results of ANOVA, there was a statistically significant relationship between lifestyle and education level $(\mathrm{P}=0.027)$. In other words, with increasing levels of education, lifestyle status also improved. T-test results showed that there was statistically significant difference between overall lifestyle mean score of elderly people with osteoporosis and those suffering from chronic disease $(\mathrm{P}<0.009)$. In other words, the mean scores of individuals with chronic disease are lower than that of the non-affected patients.

\section{Conclusion}

Osteoporosis and its complications impose heavy costs on patients and their families, especially, when it comes to aging. On the other hand, it leads to decreased quality of life in these people. Therefore, patients and other community members must reduce the incidence and complications of the disease at an older age by correcting their lifestyles in order to reduce the costs of osteoporosis. Healthy lifestyles can also help improve the quality of life at old age by decreased incidence of disease and reduced complications of the disease at the community level. Given that lifestyle of few $(10.7 \%)$ of the elderly with osteoporosis is at the optimal level, this is a remarkable and contemplative issue and requires further investigations and planning by the authorities at the macro level to address the problems concerning the lifestyle of elderly people with osteoporosis.

\section{Acknowledgments}

This research was extracted from the MSc. thesis of the first author in the Department of Geriatric Nursing, School of Nursing and Midwifery, Tehran University of Medical Sciences, Tehran.

\section{Conflict of Interest}

The authors declared no conflicts of interest. 


\title{
سبك زندگَى سالمندان مبتلا به استئويروز و عوامل مرتبط بآن
}

\author{
"اسماعيل فلاح مهرآبادى'، مينو باك كوهر '، صديقه اسدى'، حميد حقانى"

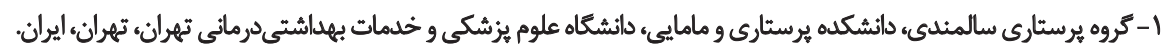

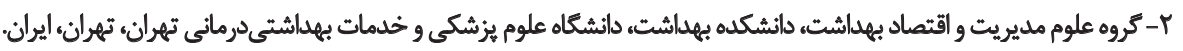

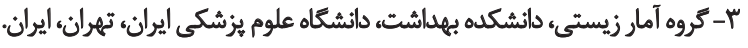

\begin{abstract}
حكيد

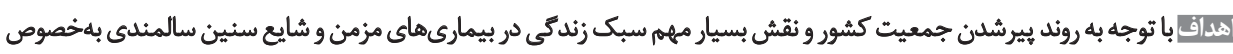

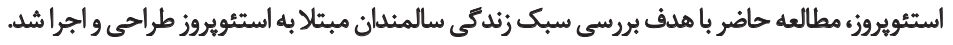

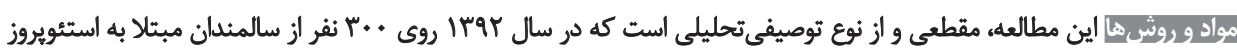

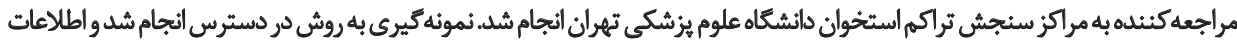

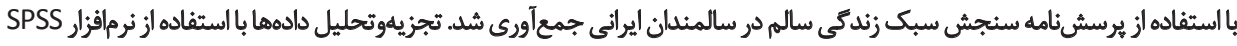

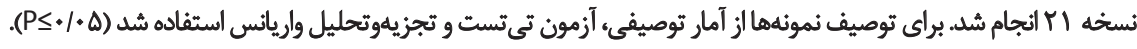

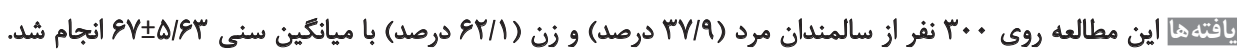

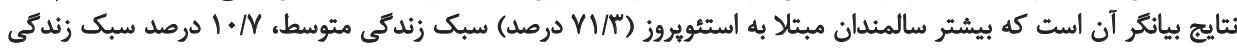

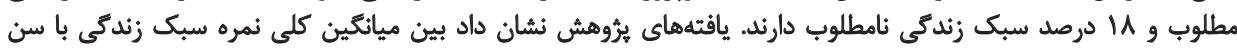

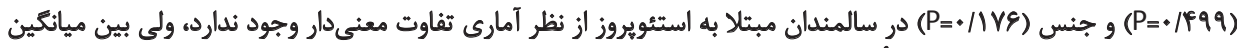

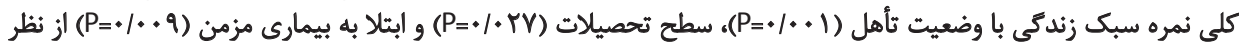

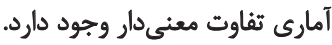

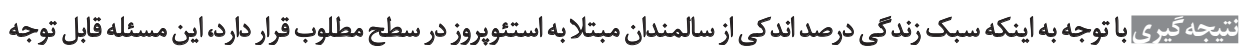

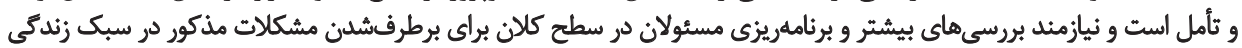
سالمندان مبثلا به استئويروز است برست.
\end{abstract}

تاريخ دريافت: • (1 بهمن هوبا

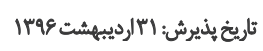

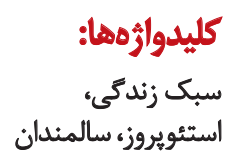

كليدوازٔها:

استئويروز، سالمندان

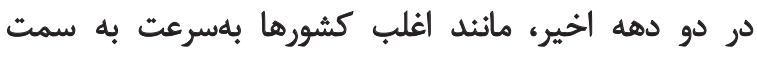

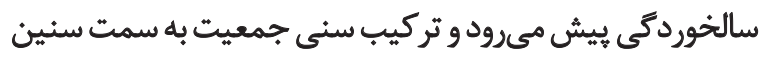

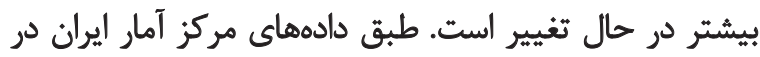

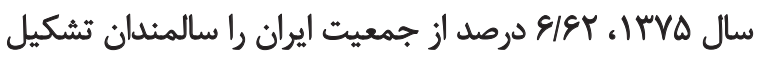

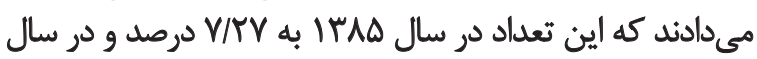

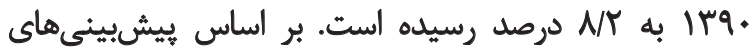

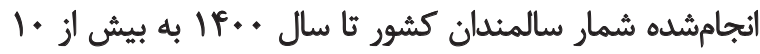

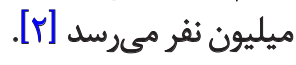

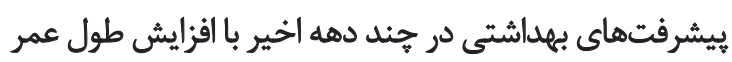

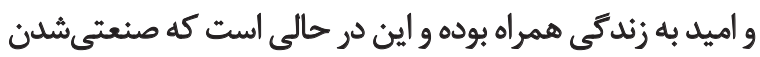

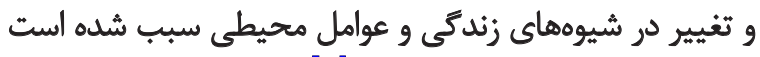

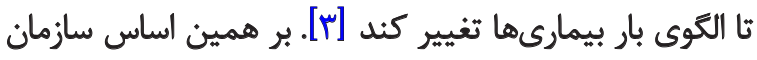

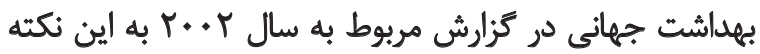

dalê

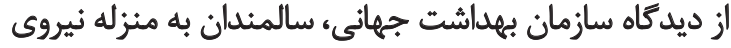

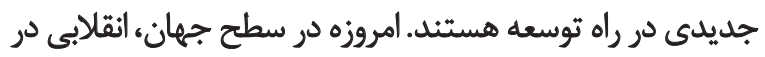

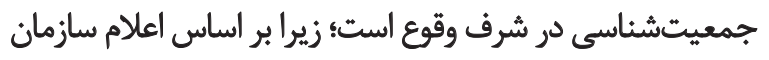

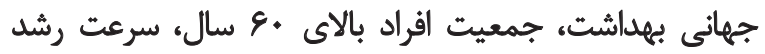

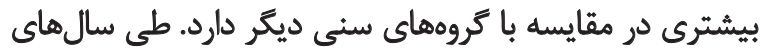

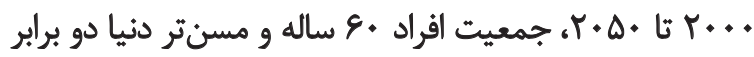

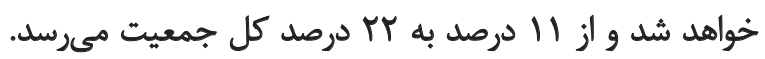

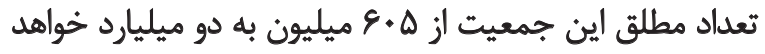

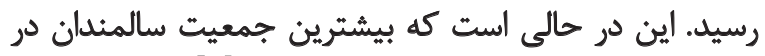

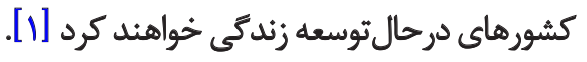
كشور مانيز يس ازطي تغييرات وسيع وسريع جمعيتشناختي 
مي شود و درنهايت كيفيت زندكى افراد مبتلا را مختل مى كند

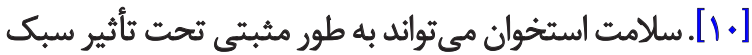

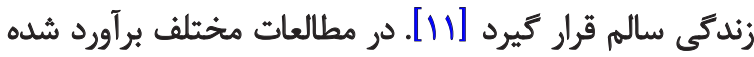

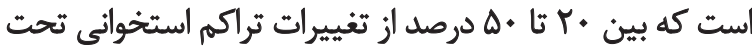

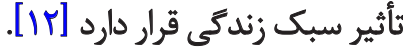

"اسبك زندكى، طبق تعريف سازمان جهانى بهداشت فعاليت

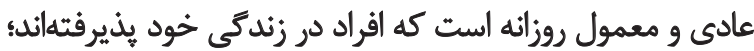

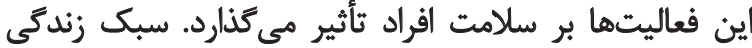

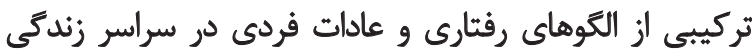

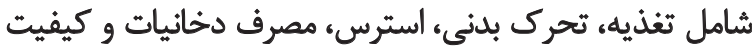

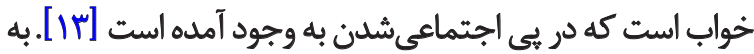

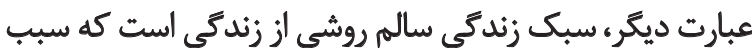

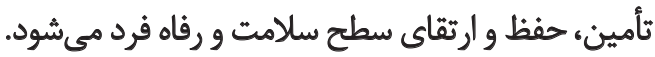

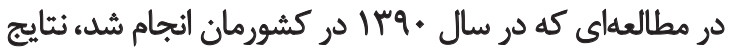

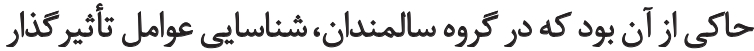

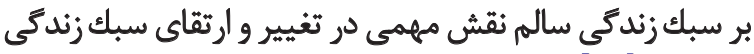

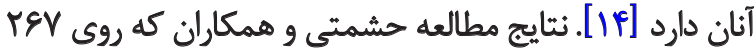

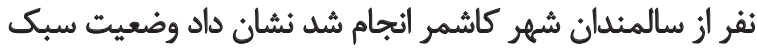

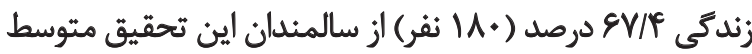

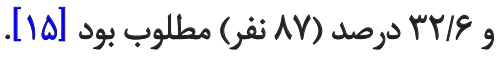

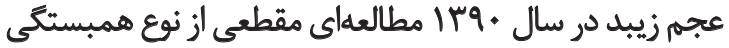

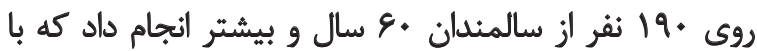

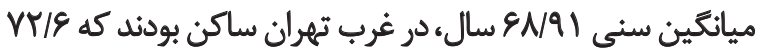

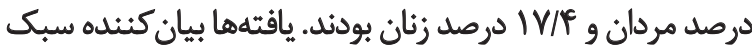

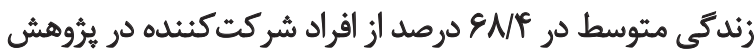

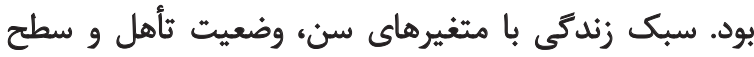

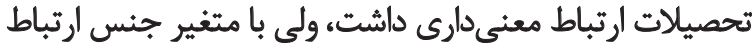

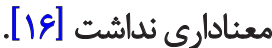

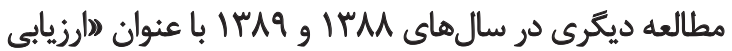

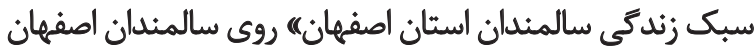

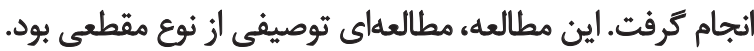

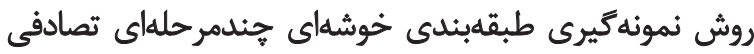

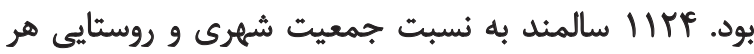

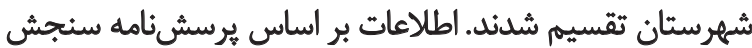

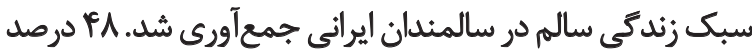

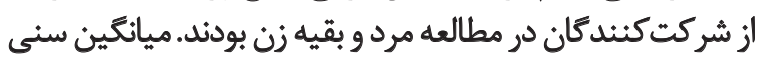

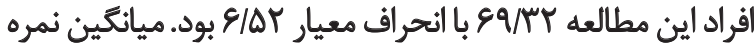

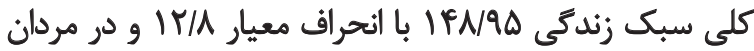

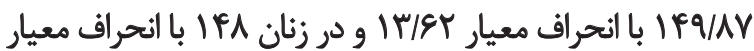

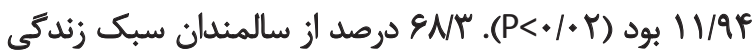

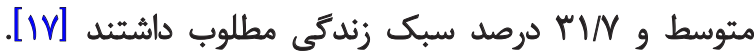

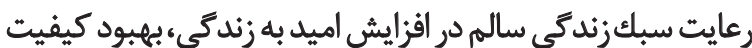

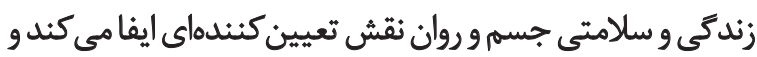

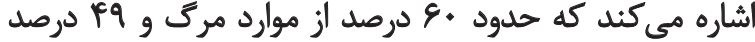

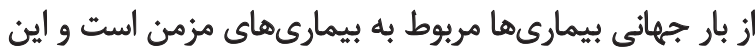

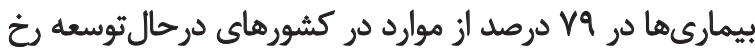

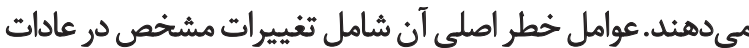

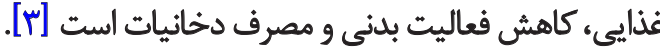
استئويروز 'نوعى بيمارى مزمن و شايعترين بيماري متابوليك

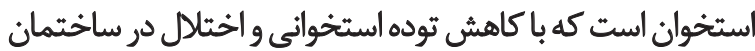

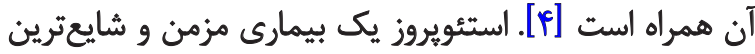

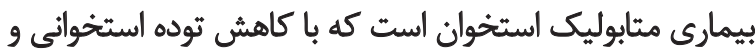

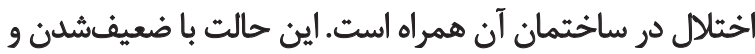

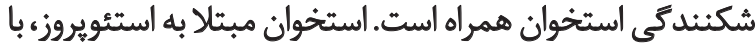

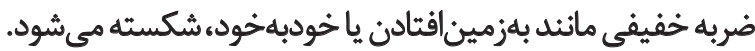
محل هاى شايع شكستَى ناشى از استئويروز شامل استخوان هاى

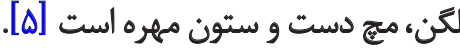
مركز تحقيقات استئويروز ايران كزارش داد كه با توجه به اينكه

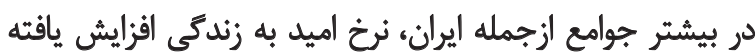

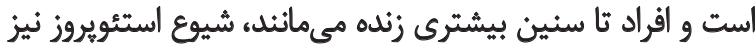

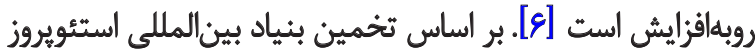

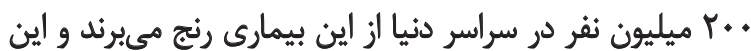

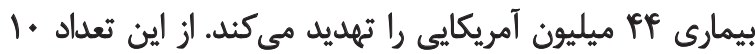

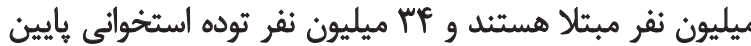

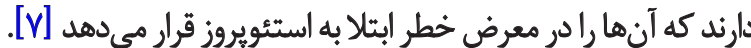

آخرين آمار موجود در ايران دال بر شيوع استئويروز (A)

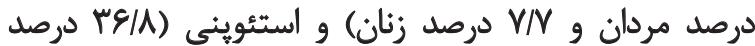

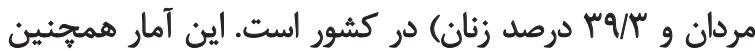

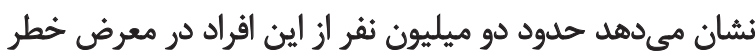

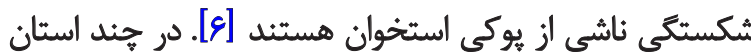

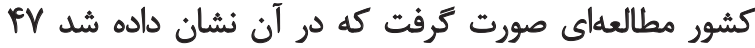

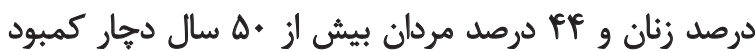

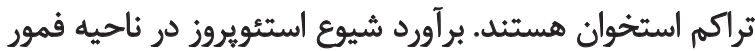

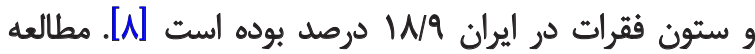

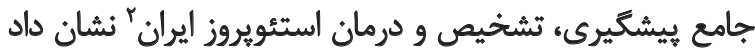

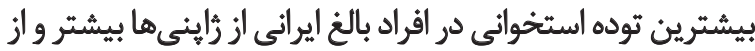

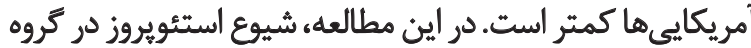

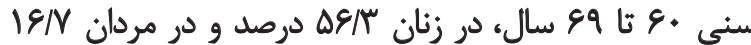

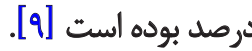

عوارض ناشى از استئويروز زندگى افراد را تحث تأثير قرار

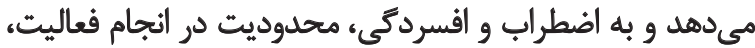

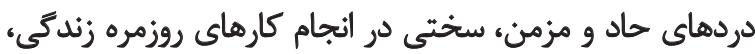

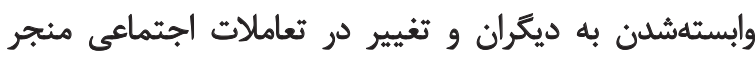

1. Osteoporosis

2. Iranian Multicenter Osteoporosis Study (IMOS) 


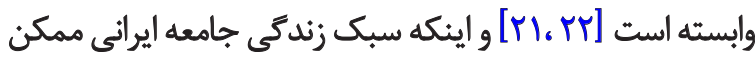

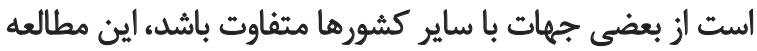

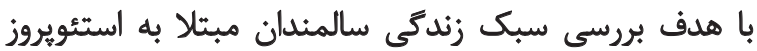

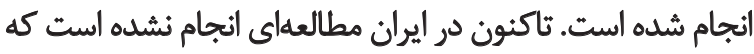

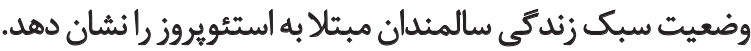

\section{روش مطالعه}

اين مطالعه، مقطعى و از نوع توصيفىتحليلى است كه روى إنى

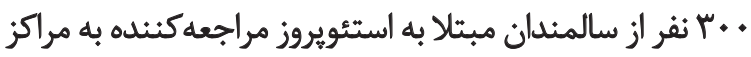

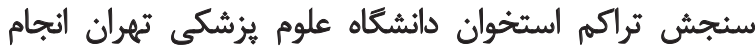

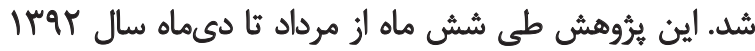

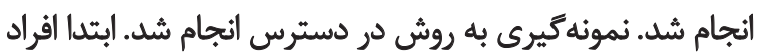

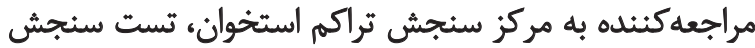

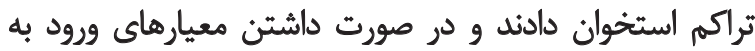

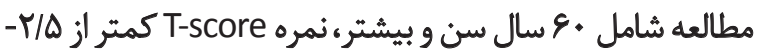

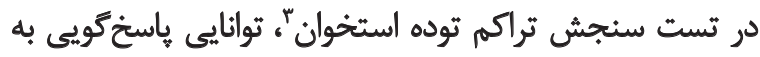

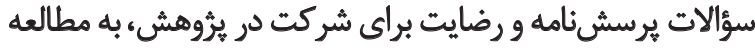

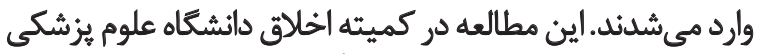

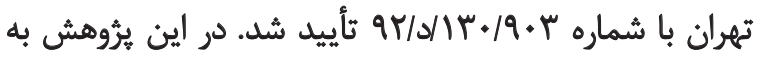

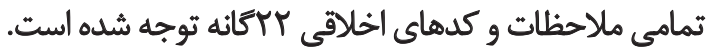
بعد از ارائه توضيحات لازم و كرفتن اجازمنامه كتبى و رضايت

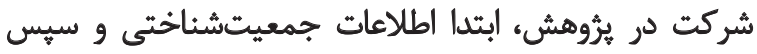

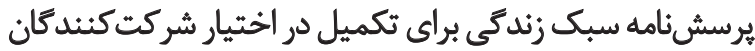

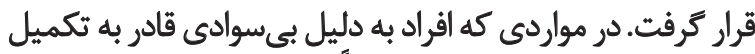

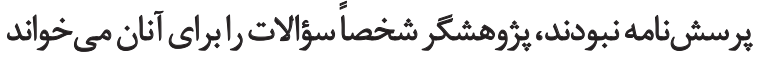

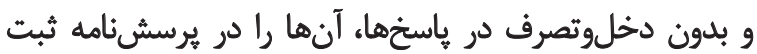

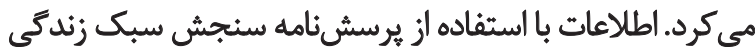

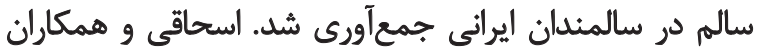

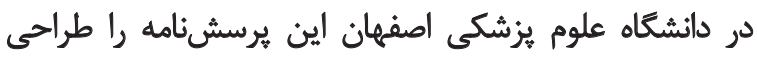

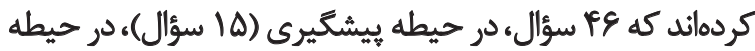

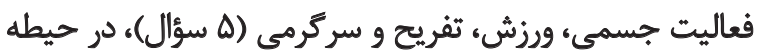

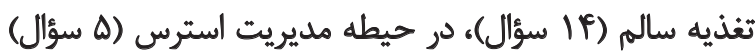

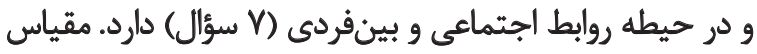

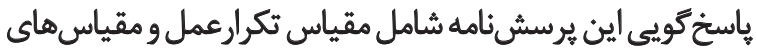

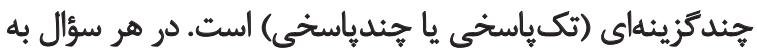

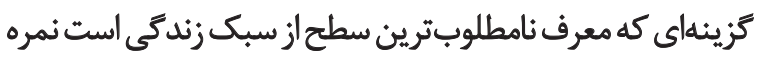

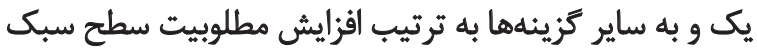

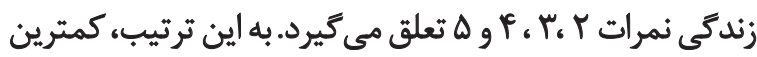

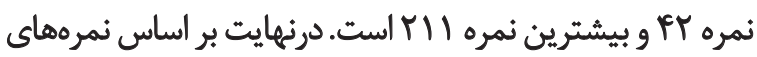

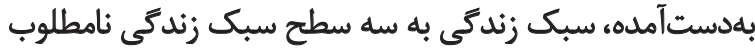

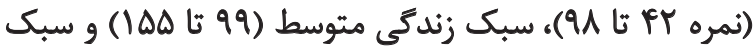

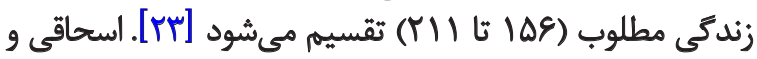

3. Bone Mass Density (BMD)

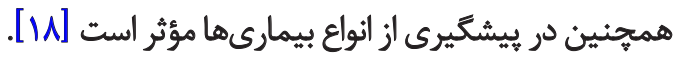
اتخاذ سبك زندگى سالم و شركت فعالانه در مراقبت از خود در

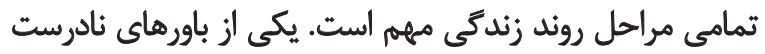

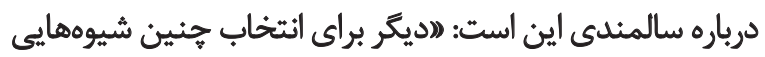

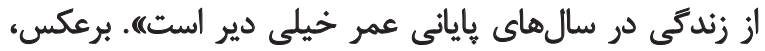

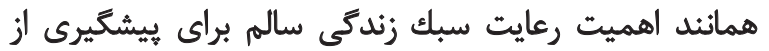

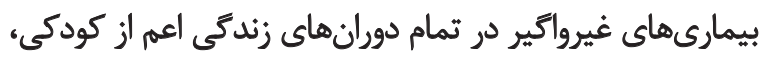

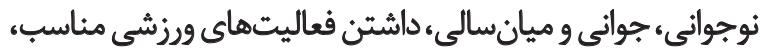

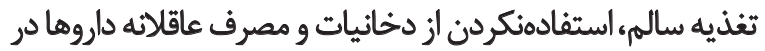

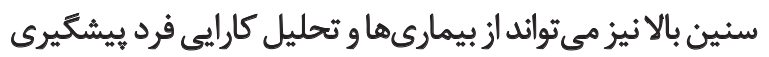

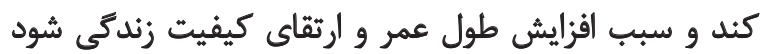

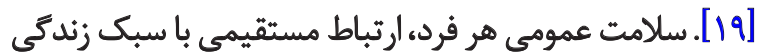

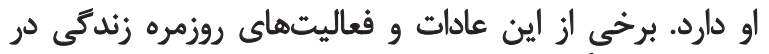

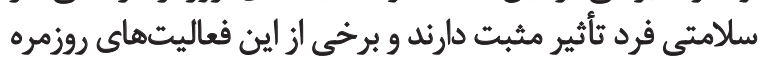

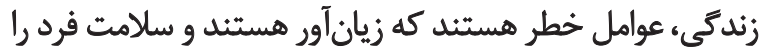

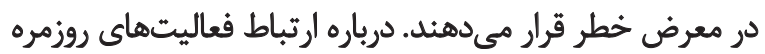

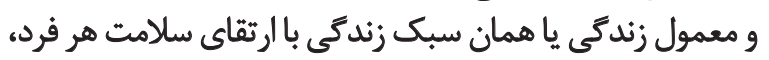

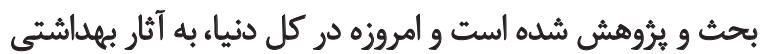
سبك زندگى توجه شده است

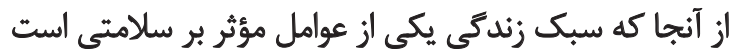

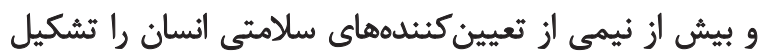

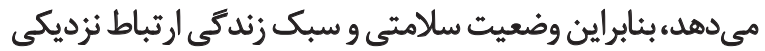

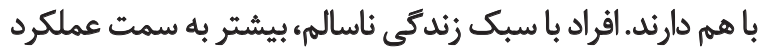

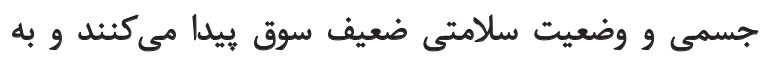

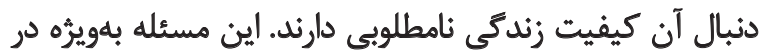

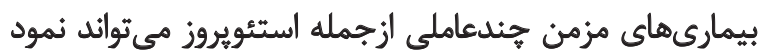

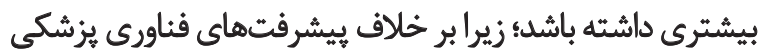

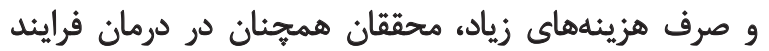

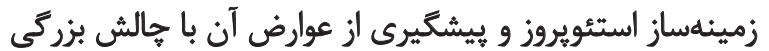

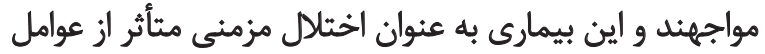

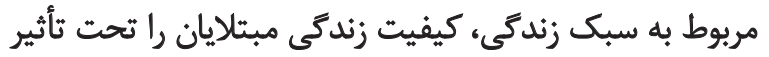

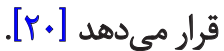

با توجه به مسائل يادشده، درمىيابيم كه رعايتنكردن سبك

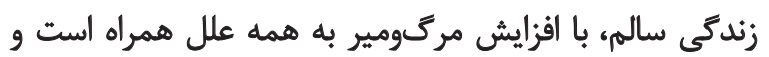

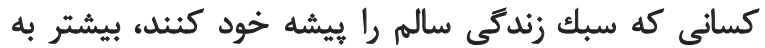

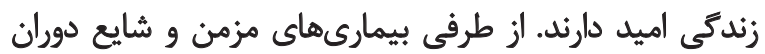

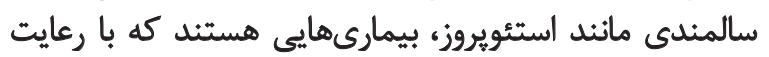

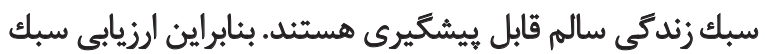

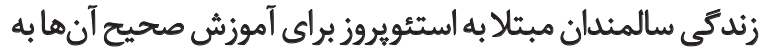

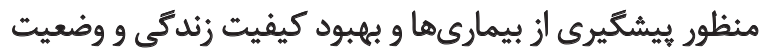
سلامتىشان اهميت زيادى دارد. با درنظركرفتن اين نكته كه سبك زندكى به فرهنك مردم 


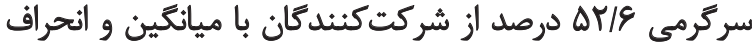

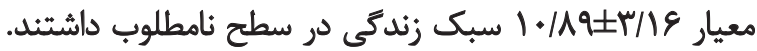

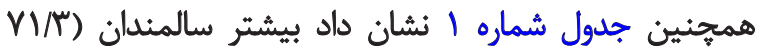

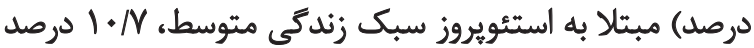

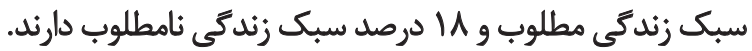

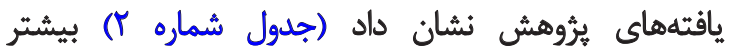

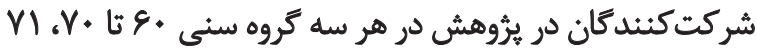

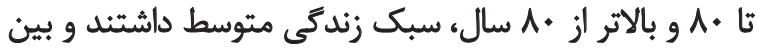

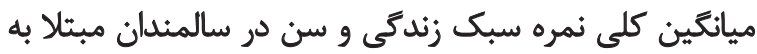

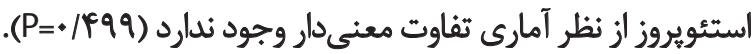
نتايج آزمون تى تست (جدول شماره "َ) نشان داد ميانكين نمره

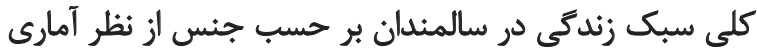

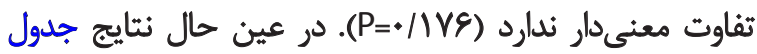

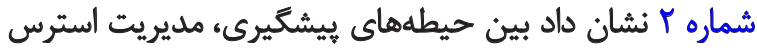

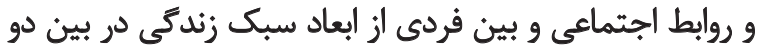

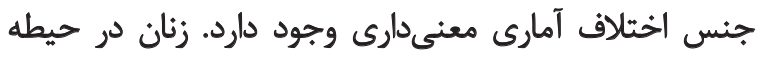

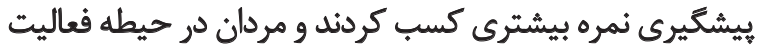

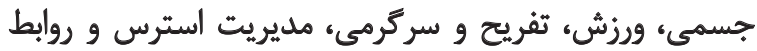
اجتماعى و بينفردى نمره بهترى كسب كردئ مكريتد.

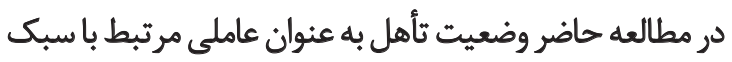

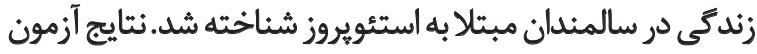

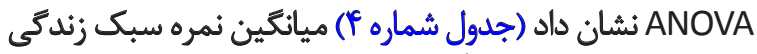

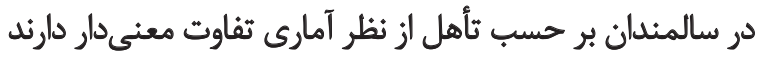

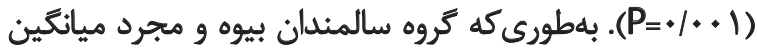

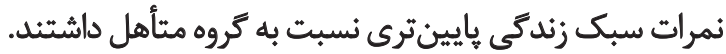
نتايج جدول شماره ه نشان داد با افزايش سطح تحصيلات،

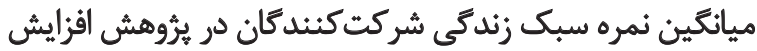

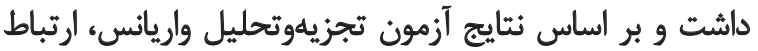

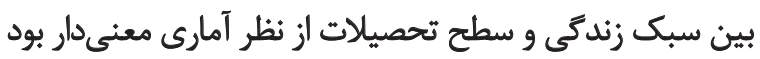

همكاران در دانشعاه علوم يزشكى اصفهان قبلاً روايى و واياييى

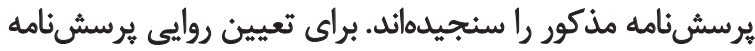

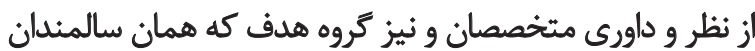

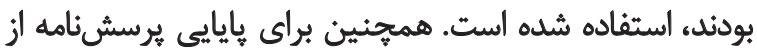

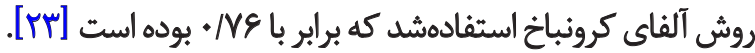

تجزيهوتحليل دادها با استفاده از نرمافزار SPSS نسخه

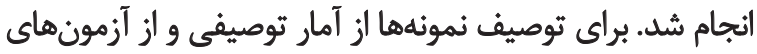

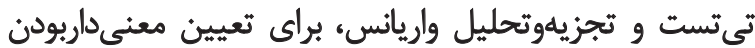

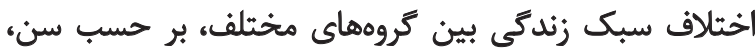

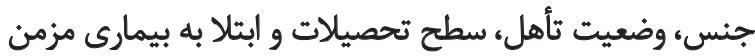

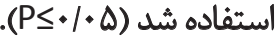

يافتهها

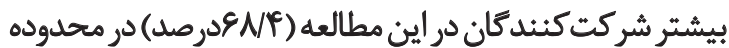

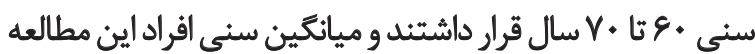

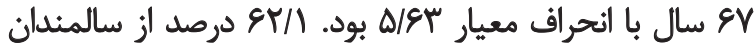

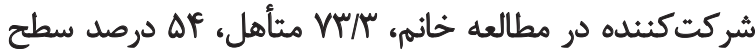

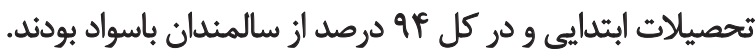

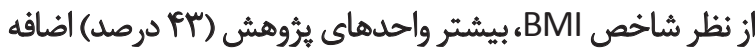

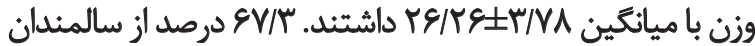

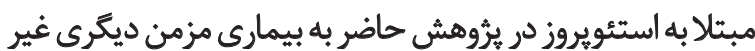

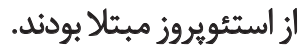

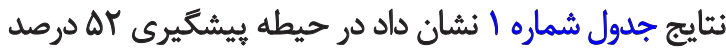
با ميانكين و انحراف معيار

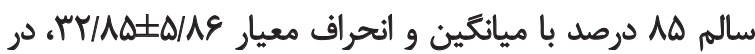

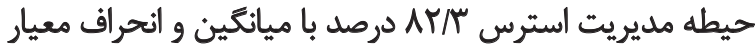
VT/\& IF/MAT/\&\&

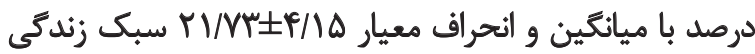
در سطح متوسط داشتند. همجنين در حيطه ورزش، تفريح و

جدول ا. توزيع فراواني مطلق و نسبيى واحدهاي يرؤهش شده بر حسب وضعيت سبك زندكى و ابعاد آن

\begin{tabular}{|c|c|c|c|c|c|c|c|c|c|c|c|c|}
\hline \multicolumn{2}{|c|}{ بين فردى اجتماعى و } & \multicolumn{2}{|c|}{ مديريث استرس } & \multicolumn{2}{|c|}{ ثغذيه سالم } & \multicolumn{2}{|c|}{ 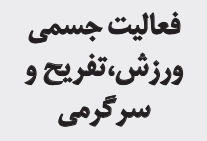 } & \multicolumn{2}{|c|}{ يشئكيرى } & \multicolumn{2}{|c|}{ سبك زندكّى كل } & \multirow{2}{*}{ زئدئى سيك } \\
\hline مرصد & فواواني & ورصد & فراوائي & درصد & فراوانى & درصد & فراوانى & مرصد & فراواتى & درصد & فراوانيى & \\
\hline $1 \Delta / \uparrow$ & pe & $F N$ & if & $r / f$ & 1. & $8 / 1$ & M & is & iff & $1 . N$ & m & مطلوب \\
\hline$V / 8$ & MM & $A T / r$ & TFV & 10 & TAD & 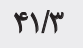 & ITF & Ar & $1 A F$ & $\mathrm{~V} / \mathrm{rr}^{\prime}$ & rif & متوسط \\
\hline ir & re & ir & $m$ & $11 / 8$ & ra & $\Delta T / E$ & $1 D A$ & - & - & M & af & نامطلوب \\
\hline \multicolumn{2}{|c|}{$r M M \pm+r / \Delta$} & \multicolumn{2}{|c|}{ 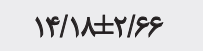 } & \multicolumn{2}{|c|}{$\Pi r / A \Delta \pm \Delta / N$} & \multicolumn{2}{|c|}{$1 . / 19 \pm m / 18$} & \multicolumn{2}{|c|}{$\Delta 1 / W \pm V / \cdot V$} & \multicolumn{2}{|c|}{ IrT/RTII\&/A. } & أتحراف معيار و ميانكين \\
\hline
\end{tabular}

记 
جدول r . سبك زئدكى واحدهاي يثروش بر حسب سن

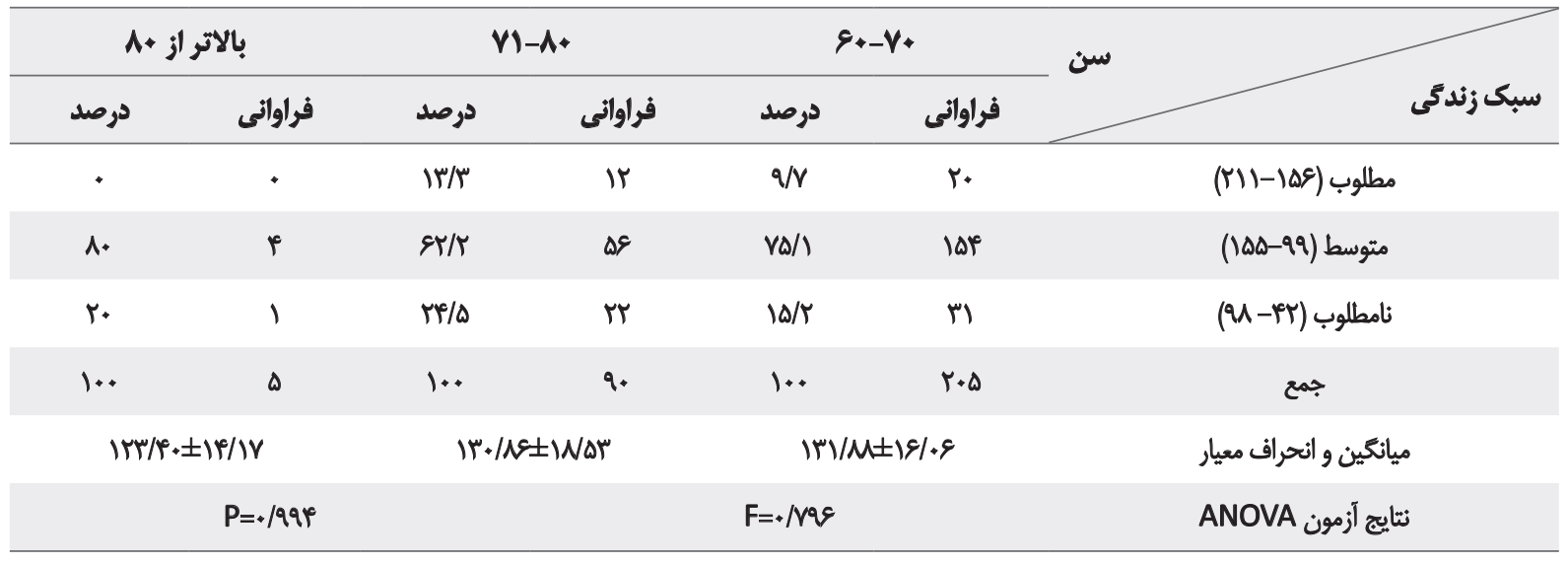

乩

جدول ". ميانكين نمره سبك زندكى و ابعاد آن در واحدهاى بيروهش بر حسب جنس

\begin{tabular}{|c|c|c|c|c|c|c|}
\hline \multicolumn{7}{|c|}{ نمره سبك زندكى } \\
\hline وروابط إجتماعي و فردى & مديريت استرس & تغغذيه سالم & فعاليت جسمى ورزئشئفريع & بيشئيرى & كل ال & جنس \\
\hline$r r / \Delta S \pm r / W$ & $\mid f / V q \pm T / R \Delta$ & $m / \circ 1 \pm f / \Delta q$ & $\| /{ }^{\mu} \mid \pm r / \cdot V$ & $e q / \cdot q \pm N \cdot \Delta$ & IrT/Fg士IF/TV & 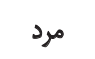 \\
\hline TIRTEYTRQ & Ir/ArET/Nq & $\pi r / r e \pm s / F$ & $1 . / 8 x \pm r / 19$ & $\Delta H / P \backslash \pm \Delta / A P$ & $\mid r q / v \Delta \pm I V / \Delta E$ & زن \\
\hline$r M M r \pm \varphi / / \Delta$ & $\mid \varphi / M \pm Y / \& S$ & $M r / \Lambda \Delta \pm \Delta / \Lambda$ S & $1 . / \Delta q \pm r / 18$ & $\Delta \mathrm{M} / \mathrm{W} \pm \mathrm{V} / \cdot \mathrm{V}$ & $|r| r|F \pm| \varepsilon / \Lambda$. & كل - تل \\
\hline $\begin{array}{l}P<+/ * \& \\
t=r / M f \Delta \\
d f=r q\end{array}$ & $\begin{array}{l}P<+/+* r \\
t=r / u r \\
d f=r q u\end{array}$ & $\begin{array}{l}P<+/ \cdot \Delta f \\
t=-1 / q m \\
d f=r q u\end{array}$ & $\begin{array}{l}P<+/ \cdot r \\
t=1 / A \cdot \Delta \\
d f=r u\end{array}$ & $\begin{array}{c}P<\cdot / *+1 \\
t=-\Delta / r e \mid \\
d f=r q\end{array}$ & $\begin{array}{c}P<+/ \neg V q \\
t=-1 / r \Delta V \\
d f=r q\end{array}$ & كَ تَمون \\
\hline
\end{tabular}

乩

نمره كلى سبك زندكَى سالمئدان ميتالا به استئوبروز با ابتلا به ميه

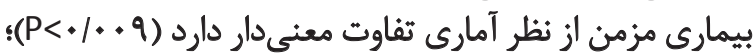

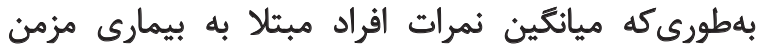

سبت (P=. PV) سبك زندكى نيز بهبود يافت. نتايج آزمون تى تست (جدول شماره ع) نشان داد ميانكين

جدول P. سبك زندكى واحدهاى برؤهش بر حسب وضعيت تأهل

\begin{tabular}{|c|c|c|c|c|c|c|c|c|}
\hline \multicolumn{2}{|c|}{ بيوه } & \multicolumn{2}{|c|}{ مطلقه } & \multicolumn{2}{|c|}{ متأهل } & \multicolumn{2}{|c|}{ مجرد } & \multirow[b]{2}{*}{ زندكّى } \\
\hline درصد & فراواتى & مرصد & فراواتى & موصد & فراواتى & درصد & فراوانى & \\
\hline$I N$ & 1 & $I F / T$ & r & $|r /|$ & rq & • & • & مطلوب (Y (MII-1I) \\
\hline $99 / 8$ & rq & $n / r$ & 1. & v. & $10 \Delta$ & 1.0 & 1. & متوسط (9Q-9ه) \\
\hline YNV & 18 & IFR & r & $18 / 9$ & re & - & - & نامطلوب (U-PT) \\
\hline $1 .$. & $\Delta P$ & $1 .$. & if & $1 .$. & $\pi$. & $1 \ldots$ & 1. & جمع \\
\hline \multicolumn{2}{|c|}{$I r r / N q \pm I f / \pi f$} & \multicolumn{2}{|c|}{$1 M+/ \Delta \cdot \pm 1 \& / r \alpha$} & \multicolumn{2}{|c|}{$\|M / m f \pm \mid V /\|$} & \multicolumn{2}{|c|}{$\| M A \pm I / M$} & ميانكين و انحراف معيار \\
\hline \multicolumn{4}{|c|}{$\mathrm{P}=* / *+1$} & \multicolumn{4}{|c|}{$F=\Delta / M T}$. & ANOVA ثتايج آزمون ANA \\
\hline
\end{tabular}

il 
جدول ه. سبك زندكى واحدهاي يثروهش بر حسب سطح تحصيلات

\begin{tabular}{|c|c|c|c|c|c|c|c|c|c|c|}
\hline \multicolumn{2}{|c|}{ داتشكاهى } & \multicolumn{2}{|c|}{ متوسطه } & \multicolumn{2}{|c|}{ راهنمايى } & \multicolumn{2}{|c|}{ ابثدايى } & \multicolumn{2}{|c|}{ بيىسواد } & \multirow[t]{2}{*}{ سطح تحصيلات } \\
\hline درصد & فراوانى & درصد & فراواتى & درصد & فراوانى & درصد & فراوائى & درصد & فراوانى & \\
\hline re & $\varepsilon$ & $\operatorname{IPN}$ & $\Delta$ & $W / 1$ & $\gamma$ & $\wedge$ & ir & $\Delta / \Delta$ & 1 & مطلوب (SD-1I) \\
\hline Ar/ & ir & eNp & $\pi$ & $n$ & pe & $n / \Delta$ & 119 & $W / A$ & If & (100-99) (19) \\
\hline$r / 9$ & $\Delta$ & IVN & 8 & $10 / 9$ & 1. & WD & r. & $18 / N$ & r & نامطلوب (U-PT) \\
\hline $1 .$. & m & $1+$. & $m$ & $1 .$. & r & 1.0 & IEY & 1.0 & M & جمع - جم \\
\hline \multicolumn{2}{|c|}{$|r \Delta / q| \pm r Y / \cdot r$} & \multicolumn{2}{|c|}{$1 r \Delta / r+ \pm m / r q$} & \multicolumn{2}{|c|}{$\mid r \Delta / \cdot c \pm 1 \% / / r$} & \multicolumn{2}{|c|}{$\mid r q / 11 \pm 1 \Delta / m$} & \multicolumn{2}{|c|}{$\|r E M \pm\| / N$} & ميائكين و انحراف معيار \\
\hline \multicolumn{4}{|c|}{$\mathrm{P}=\cdot / \cdot \mathrm{rV}$} & & & \multicolumn{2}{|r|}{$F=r(W I$} & & & نتايج آزمون ANOVA \\
\hline
\end{tabular}

L

جدول و. سبك زندكى واحدهاي بئوهش بر حسب ابثلا به بيمارى مزمن

\begin{tabular}{|c|c|c|c|c|c|}
\hline \multicolumn{2}{|c|}{ خير } & \multicolumn{2}{|c|}{ 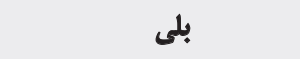 } & \multirow[t]{2}{*}{ إبتالا به بيمارى مزمين } & \multirow{2}{*}{ سبك زندكى } \\
\hline درصد & فراوانى & درصد & فراوانى & & \\
\hline $1 . / r$ & 1. & $1 . / 9$ & rr & مطلوب (III-1I) & \\
\hline $8 \Delta / r^{\prime}$ & ap & $n \in / r$ & 10. & متونط (M & \\
\hline $\mathrm{r \varphi /Q}$ & ri & $16 / 9$ & r. & نامطلوب (U-PT) & \\
\hline $1 .$. & 4 & $1 .$. & $r+r$ & جمع & \\
\hline \multicolumn{2}{|c|}{$|r \Delta|+\Delta \pm$ NEq } & \multicolumn{2}{|c|}{$|T Q / S A \pm| \Phi / \Delta F$} & ميانكين و انحراف معيار & \\
\hline$t=-r / g \pi r$ & $d f=r q u$ & \multicolumn{2}{|c|}{$P=.1 \ldots 9$} & آزآمون تى تست & \\
\hline
\end{tabular}

L

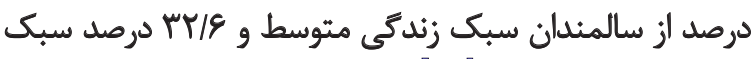

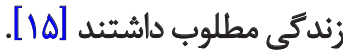

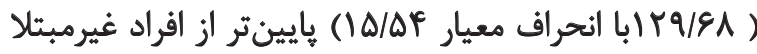

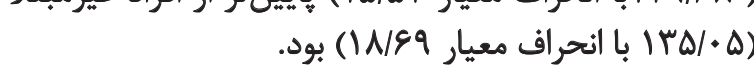

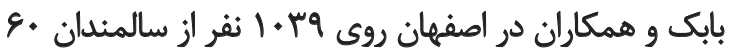

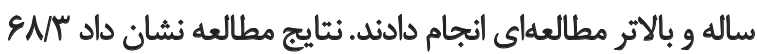

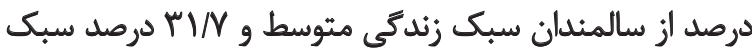

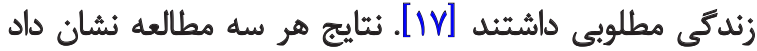

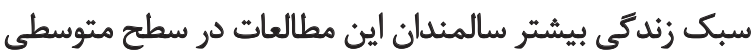

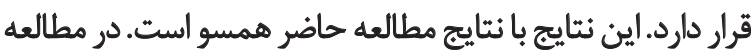

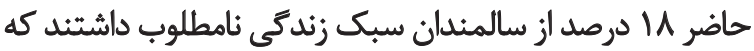

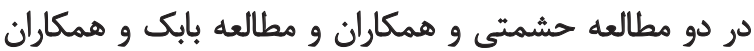

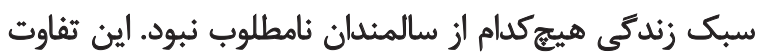

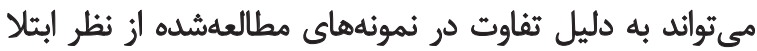

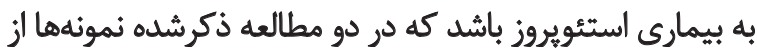

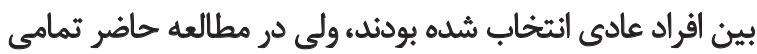

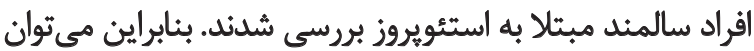
كفت كه سبك زندكى سالمندان مبتلابه استئويروز در مقايسه با بانيا

!)

هدف مطالعه حاضر، بررسى سبك زندئى در سالمندان مبتلا

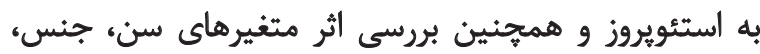

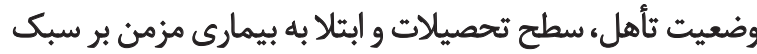

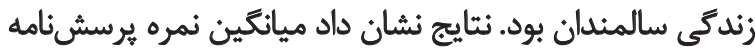

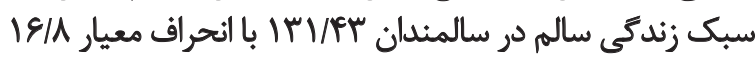

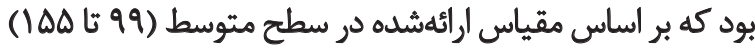

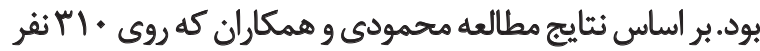

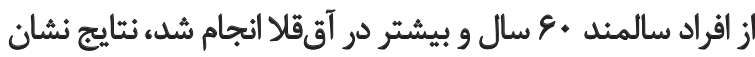

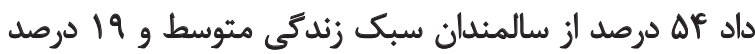

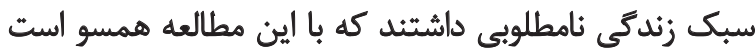

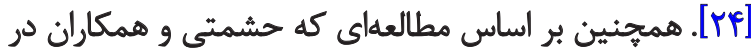

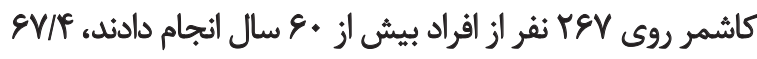


سالمندان بيوه و مجرد در مقايسه با تروه متأهل ميانتخين نمرات

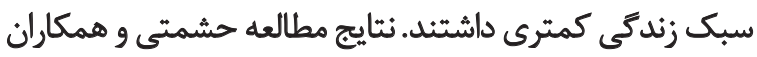

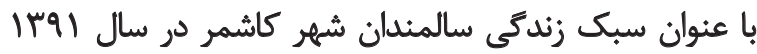

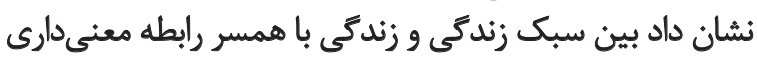

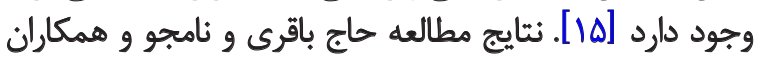

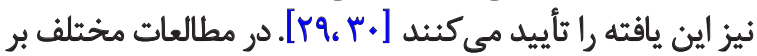

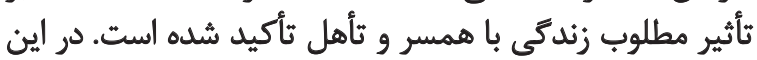

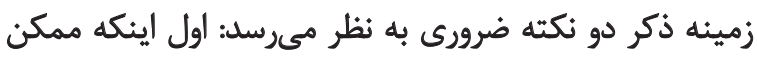

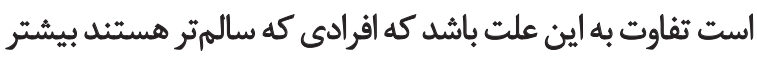

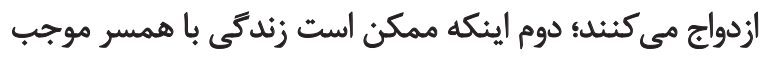

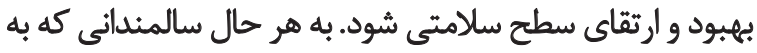

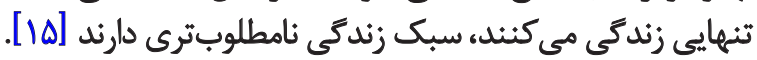

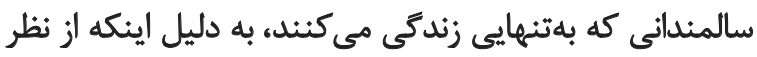

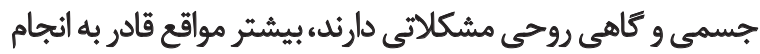

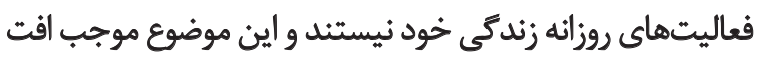

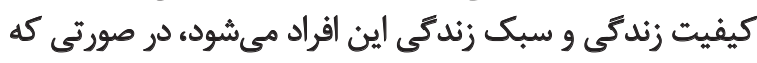

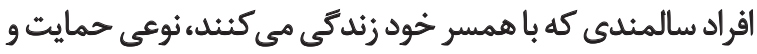

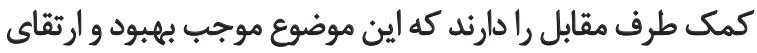

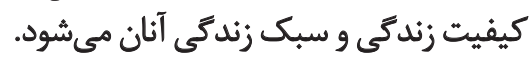

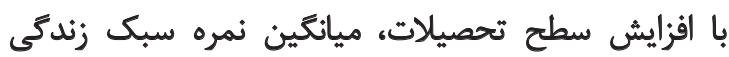

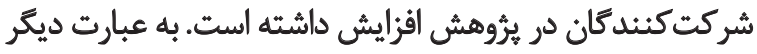

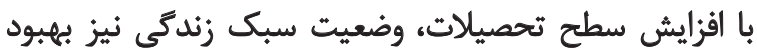

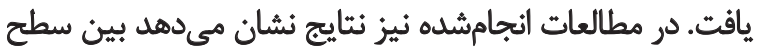

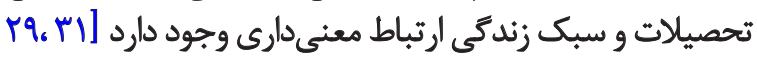
[la، IV، Yf،

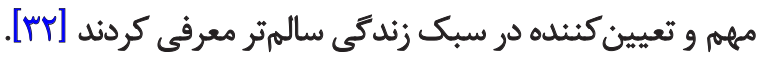

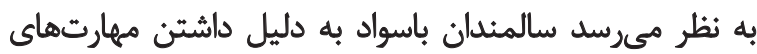

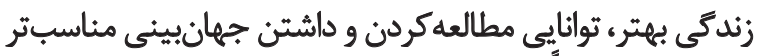

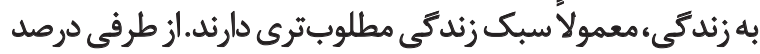

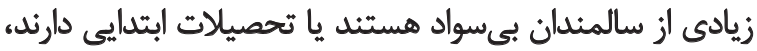

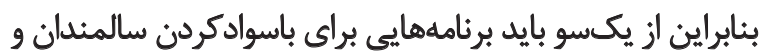

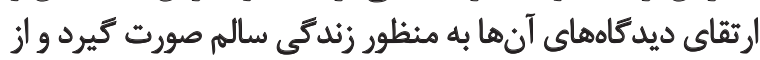

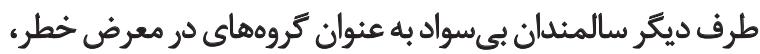

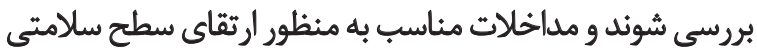

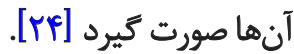

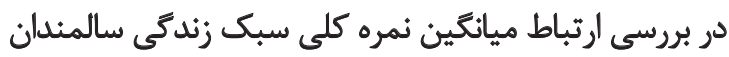

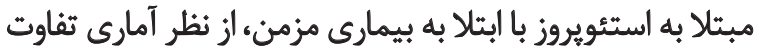

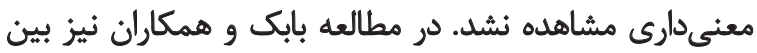

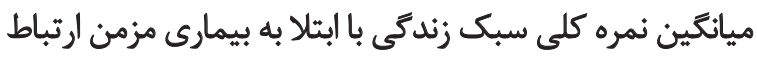

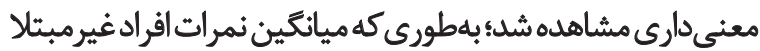

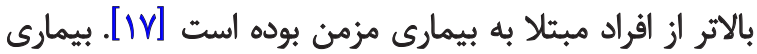

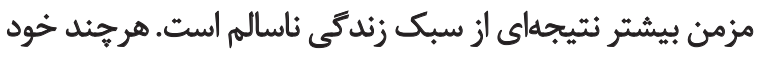

4. Hearty et al.
سالمندانى كه استئويروز ندارند، نامطلوبتر است. البته با توجه به

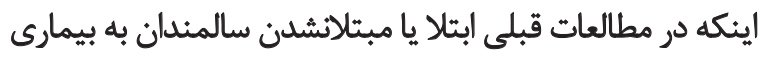

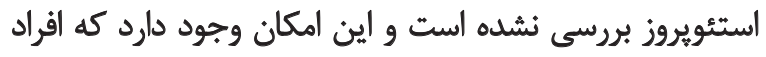

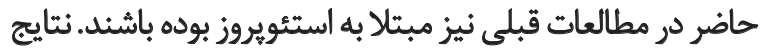

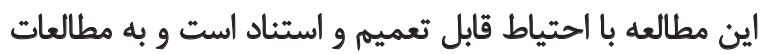

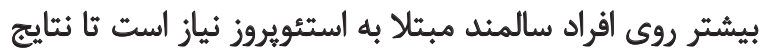

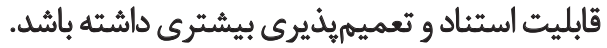

در بررسى رابطه متغير سن و سبك زندكى نتايج نشان داد

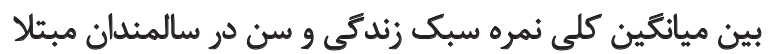

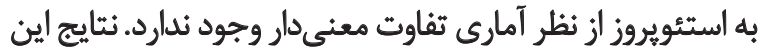

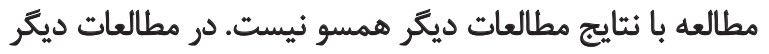

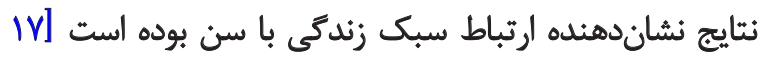

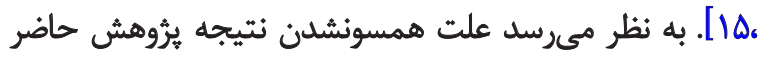

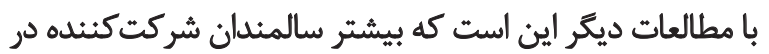

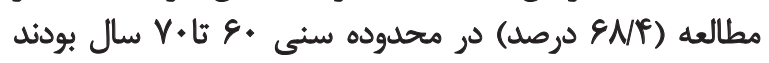

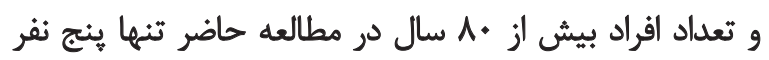

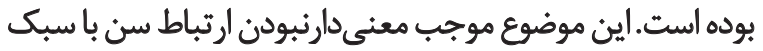
زندكى در مطالعه حاضر باشد.

بررسى ارتباط ميانكين نمره كلى سبك زئدكى در سالمندان

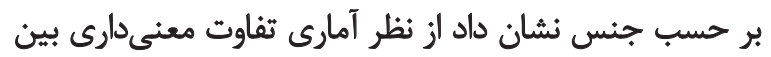

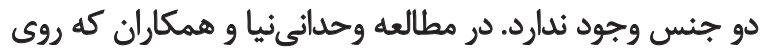

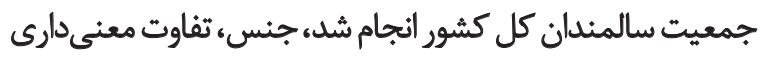

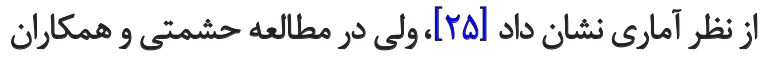

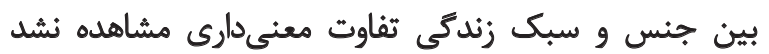

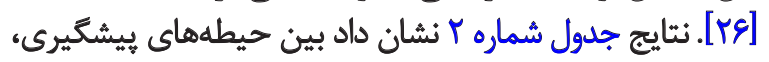

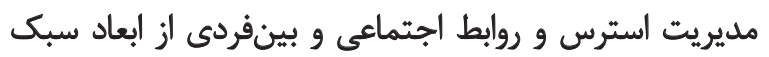

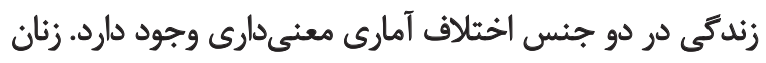

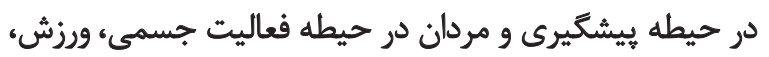

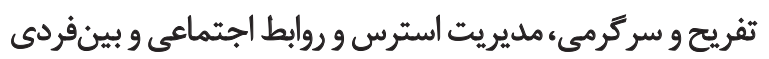

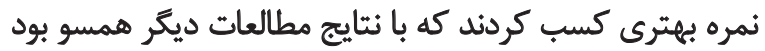

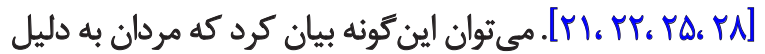

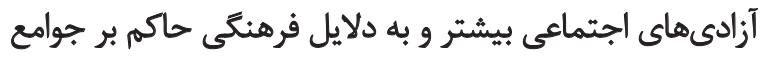

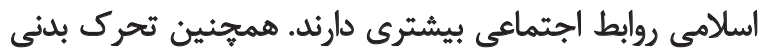

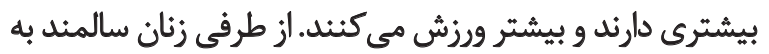

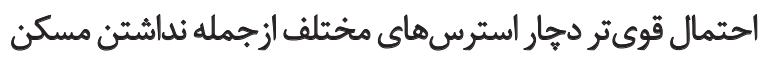

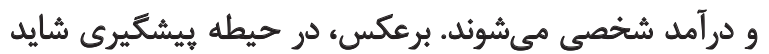

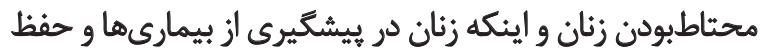

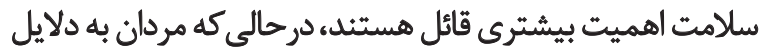

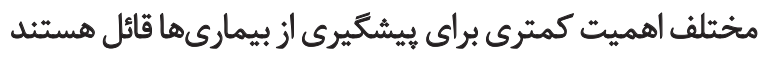

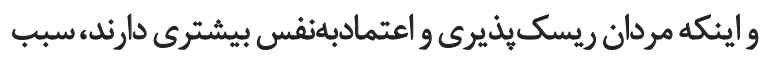

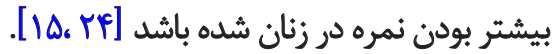

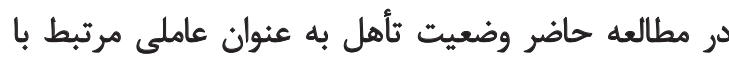

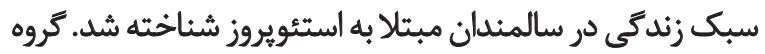


اسماعيل فلاح مهر آبادى، در گروه يرستارى سالمندى، دانشكده

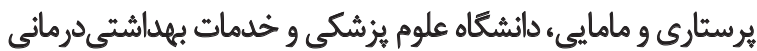

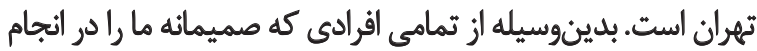

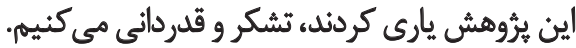

بيمارى مزمن نيز مى تواند باعث افت سبك زندكى شودن، ولى

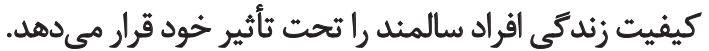

تعدادى از يرسشنامهها به روش خودَزارشدهى تكميل

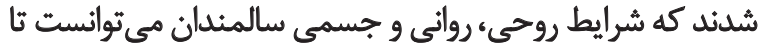

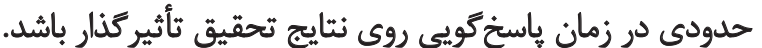

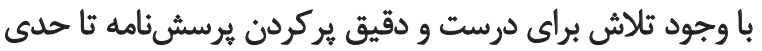
خارج از كنترل يرؤوهشك بود.

\section{تتيجهئيرىنهايى}

بيمارى استئويروز و عوارض ناشى از آن هزينههاى سنگينى را را

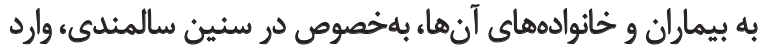

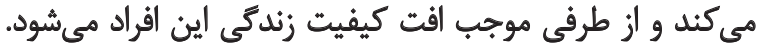

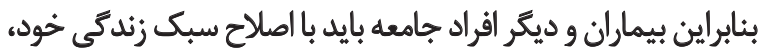

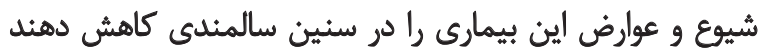

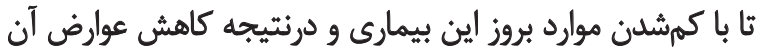

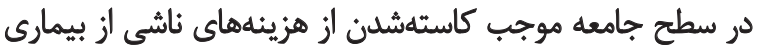

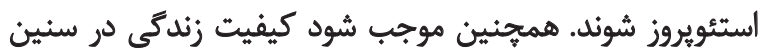

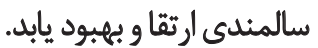

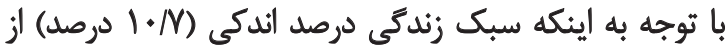

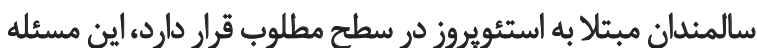

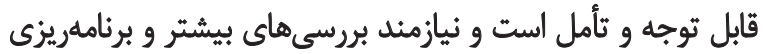

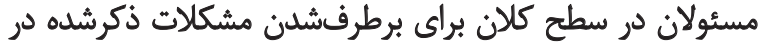
سبك زندگى سالمندان مبثلا به استئويروز است.

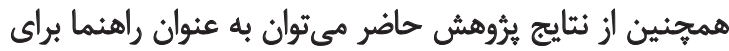

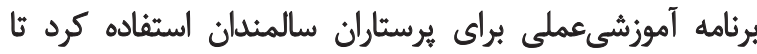

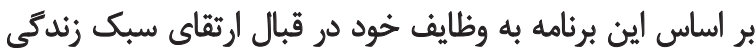

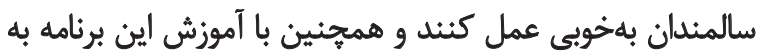

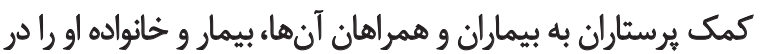
كنترل و كاهش اضطراب بيمار يارى كرد.

ارائه كنندكان خدمات سلامت به خصوص يرستار ان بهداشت جامعان

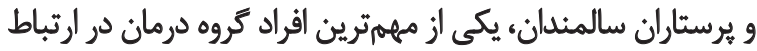

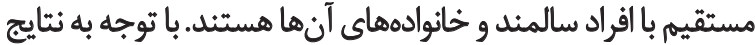

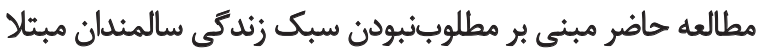

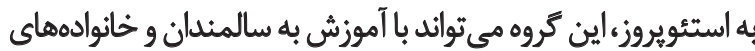

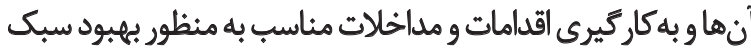

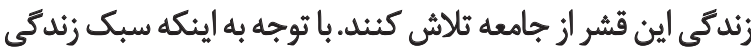

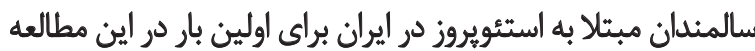

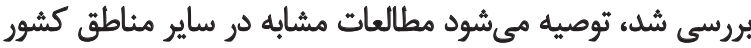
انجام گيرد ثنا نتايج حاصله قابل مقايسه باشند.

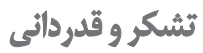

اين مطالعه بخشى از هاياننامه مقطع كارشناسى ارشد آقاى 


\section{References}

[1] World Health Organization. Health statistics and health information systems: Proposed working definition of an older person in Africa for the MDS Project. Geneva: World Health Organization; 2013.

[2] Statistical [Center of Iran. Selected population and housing census 2012 (Persian)]. Tehran: Statistical Center of Iran; 2013.

[3] World Health Organization. Increasing fruit and vegetable consumption to prevent chronic disease has profound implications for global food production. Geneva: World Health Organization; 2003.

[4] Cortet B, Blotman F, Debiais F, Huas D, Mercier F, Rousseaux $C$, et al. Management of osteoporosis and associated quality of life in post menopausal women. BMC Musculoskeletal Disorders. 2011; 12:7. doi: 10.1186/1471-2474-12-7

[5] Burge R, Dawson-Hughes B, Solomon DH, Wong JB, King $\mathrm{A}$, Tosteson A. Incidence and economic burden of osteoporosis-related fractures in the United States, 2005-2025. Journal of Bone and Mineral Research. 2007; 22(3):465-75. doi: 10.1359/jbmr.061113

[6] Osteoporosis Research Center. [Osteoporosis (Persian)] [Internet]. 2011. [Cited 2012 October 5]. Available from: http:// emri.tums.ac.ir/pages/mainpage.asp?I=S10M5P2C1

[7] National Osteoporosis Foundation. Osteoporosis: review of the evidence for prevention, diagnosis and treatment and cost-effectiveness analysis. Osteoporosis International. 1998; 8(S4):S7-S80 doi: 10.1007/pl00022721

[8] Bagheri P, Haghdoost AA, Dortaj rabari E, Halimi L, vafaei $Z$, farhang nya $M$, et al. [Ultra analysis of prevalence of osteoporosis in Iranian women a systematic review and meta-analysis (Persian)]. Iranian Journal of Endocrinology and Metabolism. 2011; 13(3): 315-325.

[9] Shirazi Khah M. [Study of health and social indicators of elderly women in Iran (Persian)]. Iranian Journal of Ageing. 2012; 6(S1):66-78

[10] Johnell O, Kanis J. Epidemiology of osteoporotic fractures. Osteoporosis International. 2004; 16(S2):3-7. doi: $10.1007 /$ s00198-004-1702-6

[11] Dorner T, Weichselbaum E, Lawrence K, Viktoria Stein K, Rieder A. Austrian osteoporosis report: epidemiology, lifestyle factors, public health strategies. Wiener Medizinische Wochenschrift. 2009; 159(9-10):221-9. doi: 10.1007/s10354009-0649-9

[12] Morgan SL. Calcium and Vitamin D in Osteoporosis. Rheumatic Disease Clinics of North America. 2001; 27(1):101-30. doi: 10.1016/s0889-857x(05)70189-7

[13] Shaw JW, Johnson JA, Coons SJ. US valuation of the EQ-5D health states. Medical Care. 2005; 43(3):203-20. doi: 10.1097/00005650-200503000-00003

[14] Taghdici MH, Doshmangir P, Dehdari T, Doshmangir L. Influencing factors on healthy lifestyle from viewpoint of elderly people: Qualitative study. Iranian Journal of Ageing. 7(4):47-58

[15] Heshmati H, Asnashari R, Khajavi S, Charkazi A, Babak A, Hosseini SG, et al. [Life style of elderly in Kashmar, Iran
2012 (Persian)]. Journal of Research Development in Nursing \& Midwifery. 2014; 11(1):39-50.

[16] Ajamzibod H. [The study of relationship between life style and quality of life among the west Tehran elderly (Persian)] [MSc. thesis]. Tehran: Tehran University of Medical Sciences.

[17] Babak A, Davari S, Aghdak P, Pirhaji O. [Assessment of healthy lifestyle among elderly in Isfahan, Iran (Persian)]. Journal of Isfahan Medical School. 2011; 29(149):1-11.

[18] Suzuki M, Ohyama N, Yamada K, Kanamori M. The relationship between fear of falling, activities of daily living and quality of life among elderly individuals. Nursing and Health Sciences. 2002; 4(4):155-61. doi: 10.1046/j.14422018.2002.00123.x

[19] Dolan P. Modeling valuations for EuroQol health states. Medical Care. 1997; 35(11):1095-108. doi: 10.1097/00005650199711000-00002

[20] Silverman SL, Minshall ME, Shen W, Harper KD, Xie S. The relationship of health-related quality of life to prevalent and incident vertebral fractures in postmenopausal women with osteoporosis: Results from the Multiple Outcomes of Raloxifene Evaluation Study. Arthritis \& Rheumatism. 2001; 44(11):2611-9. doi: 10.1002/1529-0131(200111)44:11<2611::aid-art441>3.0.co;2n

[21] Muraki S, Yamamoto S, Ishibashi H, Oka H, Yoshimura $\mathrm{N}$, Kawaguchi H, et al. Diet and lifestyle associated with increased bone mineral density: cross-sectional study of Japanese elderly women at an osteoporosis outpatient clinic. Journal of Orthopaedic Science. 2007; 12(4):317-20. doi: $10.1007 / \mathrm{s} 00776-007-1143-0$

[22] Gullberg B, Johnell O, Kanis JA. World-wide projections for hip fracture. Osteoporosis International . 1997; 7(5):40713. doi: $10.1007 / \mathrm{pl} 00004148$

[23] Eshaghi SR, Farajzadegan Z, Babak A. [Healthy lifestyle assessment questionnaire in elderly: Translation, reliability and validity (Persian)]. Payesh. 2010; 9(1):91-9.

[24] Mahmudi G, Niazazari K, Sanati T. [Evaluation of life style in the elderly (Persian)]. Journal of Health Breeze. 2013; 1(3):45-50

[25] Vahdani Nia M, Goshtasebi A, Montazeri A, Maftoon F. [Health-related quality of life in an elderly population in Iran: A population-based study (Persian)]. Payesh. 2005; 4(2):113-20.

[26] Heshmati H, Behnampour N, Charkazi A, Asadi Z Dehnadi A. [Elderly hygiene status in rural areas of Golestan Province in Iran (Persian)]. Iranian Journal of Ageing. 2012; 7(1):25-33.

[27] Hanioka T, Ojima M, Tanaka K, Aoyama H. Association of total tooth loss with smoking, drinking alcohol and nutrition in elderly Japanese: Analysis of national database. Gerodontology. 2007; 24(2):87-92. doi: 10.1111/j.17412358.2007.00166.x

[28] Ueno M, Ohara S, Inoue M, Tsugane S, Kawaguchi Y. Association between education level and dentition status in Japanese adults: Japan public health center-based oral health study. Community Dentistry and Oral Epidemiology. 2012; 40(6):481-7. doi: 10.1111/j.1600-0528.2012.00697.x 
[29] Adib-Hajbaghery M, Akbari H. [The severity of old age disability and its related factors (Persian)]. Feyz. 2009; 13(3):225-34.

[30] Namjoo A, Niknami M, Baghaee Mozhgan SSM, Atrkar Roshan Z. [Survey health behavior of elderly referring to retirement centers in Rasht city in 2009 (Persian)]. Journal of Holistic Nursing and Midwifery. 2010; 20(63):40-49.

[31] Najimi A, Goudarzi AM. [Healthy lifestyle of the elderly: A cross-sectional study (Persian)]. Health System Research. 2011; 8(4):581-7.

[32] Hearty ÁP, McCarthy SN, Kearney JM, Gibney MJ. Relationship between attitudes towards healthy eating and dietary behaviour, lifestyle and demographic factors in a representative sample of Irish adults. Appetite. 2007; 48(1):1-11. doi: 10.1016/j.appet.2006.03.329 
